\title{
PROCESSO DE ELETROCOAGULAÇÃO-FLOTAÇÃO: INVESTIGAÇÃO DOS PARÂMETROS OPERACIONAIS PARA O TRATAMENTO DE ÁGUAS RESIDUAIS DA INDÚSTRIA DE PESCADOS
}

\author{
Jair J. João ${ }^{a}, *$, Tuane Emerick ${ }^{a}$, Urias de S. Filho e Rafael K. Nishihora ${ }^{\mathrm{b}}$ \\ a'Departamento de Química, Universidade do Sul de Santa Catarina, 88704-900 Tubarão - SC, Brasil \\ bDepartamento de Engenharia Química e Engenharia de Alimentos, Universidade Federal de Santa Catarina, 88040-900 Florianópolis \\ $-\mathrm{SC}$, Brasil
}

Recebido em 28/07/2017; aceito em 06/11/2017; publicado na web em 04/12/2017

\begin{abstract}
ELECTROCOAGULATION-FLOTATION PROCESS: INVESTIGATION OF OPERATIONAL PARAMETERS FOR WASTEWATER TREATMENT FROM FISHERY INDUSTRY. The objective of this study was the utilization of an electrocoagulation-flotation process to evaluate the treatment of a real wastewater from fish industries (Brazil - SC). This type of effluent is usually treated by conventional processes, which are often not sufficient to meet the desired final quality of legal environmental requirements. The effect of current density, $\mathrm{pH}$ and treatment time was evaluated. The best experimental conditions for the process were current of $5 \mathrm{~A}$, potential of $15 \mathrm{~V}$ and distance between electrodes equal to $10 \mathrm{~mm}$. Under these conditions, the removal of the contaminants were expressive for all analyzed parameters as follows: average color reduction of $95.5 \%$; turbidity of 96.9\%; COD 93.3; BOD5 of $97.5 \%$; phosphorus of $88.5 \%$ and oils and greases of $93.6 \%$.
\end{abstract}

Keywords: electrochemical process; electrocoagulation; wastewater; fish industry.

\section{INTRODUÇÃO}

O fornecimento de água limpa à população constitui um dos grandes desafios contemporâneos. O abastecimento de água potável é crítico, especialmente nos países subdesenvolvidos. ${ }^{1}$ Rios, canais e outros corpos de água são constantemente poluídos devido à descarga indiscriminada de águas residuais (efluentes líquidos). ${ }^{2}$ Países altamente desenvolvidos, como os EUA, também se defrontam com problemas relacionados ao abastecimento de água, o que por sua vez instiga o reaproveitamento de águas residuais. ${ }^{3}$ Dessa forma, cria-se uma demanda por metodologias mais eficazes e baratas para o tratamento de águas residuais diante das tecnologias tradicionais existentes. $^{4}$

Contudo, muitas vezes as propriedades ou características das águas residuais industriais variam largamente de uma indústria para outra, e também na mesma indústria, dependendo de fatores como a variabilidade da matéria-prima. ${ }^{5}$ Para a maioria das indústrias de beneficiamento, as operações produzem águas residuais que contêm contaminantes orgânicos e inorgânicos solúveis, partículas coloidais e sólidos suspensos. Dependendo do tipo de operação, o grau de contaminação pode variar largamente entre baixo, médio ou alto. ${ }^{6}$

Águas residuais industriais contaminadas por compostos orgânicos são tratadas, comumente, através de sistemas biológicos, amplamente utilizados para o tratamento de efluentes líquidos lançados nos sistemas fluviais e marinhos. ${ }^{7} \mathrm{O}$ tratamento biológico possui, entretanto, algumas desvantagens, dentre as quais se destacam a grande área ocupada pelos sistemas convencionais de tratamento biológico (várias lagoas aeróbias, anaeróbias e facultativas), a exalação de odores desagradáveis e sua total inutilidade na presença de resíduos que contenham substâncias nocivas aos microorganismos (pouco biodegradáveis ou não biodegradáveis). ${ }^{8}$ As desvantagens apresentadas justificam a investigação de novos métodos.

A indústria de beneficiamento de pescados representa um exemplo de geração de águas residuais contaminadas por compostos orgânicos. Esse setor é associado à produção de grandes volumes de efluentes,

*e-mail: jair.joao@unisul.br que apresentam alta carga de matéria orgânica, elevada turbidez, forte coloração amarelada e odor desagradável. ${ }^{9}$ As águas residuais resultam de diferentes etapas do processo, tais como: recepção, acondicionamento nas câmaras frigoríficas, evisceração/descamação, cozimento, acondicionamento nas embalagens, lavagem dos recipientes, lavagem do setor de processamento, entre outros. Neste tipo de indústria, estima-se que para cada $1,0 \mathrm{~kg}$ de produto que é processado geram-se em média 4 a 6 vezes de água contaminada. ${ }^{10}$

Diferentes metodologias são descritas na literatura para tratamento de águas residuais que apresentam as mesmas características da indústria de beneficiamento de pescados. Tratamentos físico-químicos convencionais consistem de tratamentos preliminares, como a coagulação, floculação, sedimentação e processamento do lodo. Tratamento secundário e terciário com filtros biológicos e lodos ativados também podem ser empregados. ${ }^{11}$ Contudo, esses processos não são muito eficientes quando o efluente apresenta baixo conteúdo de partícula coloidal suspensa e alta carga de matéria orgânica.

Uma alternativa ao tratamento mencionado é a utilização de processos eletroquímicos para a transformação total das moléculas orgânicas em gás carbônico e água. ${ }^{12}$ Tal nível de oxidação é alcançado por meio do uso de fortes agentes oxidantes gerados eletroquimicamente, que convertem os poluentes aquosos em produtos menos danosos. Para que as reações eletroquímicas ocorram, é necessário uso de materiais ou de eletrodos que favoreçam a combustão ou eletrocoagulação-flotação da matéria orgânica existente na água residual. Estes eletrodos acumulam oxigênio ativos em suas superfícies e são responsáveis pela combustão ou eletrocoagulação-flotação da matéria orgânica. ${ }^{13}$

A eletrocoagulação-flotação (ECF) consiste em um processo eletroquímico baseado na geração de agentes coagulantes e floculantes por meio de reações que ocorrem no ânodo e no cátodo (reações eletroquímica 1 a 4), substituindo os aditivos químicos que são utilizados nos processos convencionais. ${ }^{14}$ Durante o processo eletroquímico, a geração de coagulantes ocorre in situ com eletrodos de ferro e/ou alumínio submetidos a uma corrente elétrica. ${ }^{15} \mathrm{O}$ equipamento envolvido nesse processo é confiável e seguro, pois apenas baixo potencial eletroquímico é utilizado (intervalo entre 5 a 
$20 \mathrm{~V})$. Todavia, a quantidade relativa de gases produzidos depende da corrente e por vezes da salinidade da solução. ${ }^{16} \mathrm{O}$ processo de ECF é definido por dois mecanismos, conforme descrito nas reações eletroquímicas 1 a $4 .{ }^{17}$

No ânodo:

$$
\begin{gathered}
M_{(s)} \rightarrow M_{(a q)}{ }^{n+}+n e^{-} \\
2 H_{2} O_{(l)} \rightarrow 4 H_{(a q)}^{+}+O_{2(g)}+4 e^{-}
\end{gathered}
$$

No cátodo:

$$
\begin{gathered}
M_{(a q)}^{n+}+n e^{-} \rightarrow M_{(s)} \\
2 \mathrm{H}_{2} \mathrm{O}_{(l)}+2 e^{-} \rightarrow 2 \mathrm{H}_{2(g)}+2 \mathrm{OH}^{-}
\end{gathered}
$$

A ECF é uma tecnologia em evolução, pois apesar de haver diversos estudos envolvendo o tratamento de águas residuais, ${ }^{18}$ ainda persiste uma escassez de conhecimento científico considerando-se o potencial que essa técnica apresenta para o tratamento de águas residuais da indústria de beneficiamento de pescados. Assim, o objetivo deste artigo foi avaliar a eficiência do processo de ECF no tratamento de águas residuais geradas por duas indústrias de beneficiamento de pescados, ambas localizadas no Sul de Santa Catarina (Brasil).

\section{PARTE EXPERIMENTAL}

As amostras foram coletadas mensalmente, por um período de um ano, na entrada da estação de tratamento de efluentes de duas empresas de pescados, ambas localizadas no sul do Estado de Santa Catarina (Brasil). As coletas foram feitas de acordo com as recomendações da NBR 9897 - Planejamento de amostragem de efluentes líquidos e corpos receptores, ${ }^{19}$ e NBR 9898 - Preservação e técnicas de amostragem de efluentes líquidos e corpos receptores. ${ }^{20}$

\section{Características do reator}

Para realização do procedimento experimental foi construído um reator de acrílico com $30 \mathrm{~cm}$ de comprimento, $20 \mathrm{~cm}$ de altura e $15 \mathrm{~cm}$ largura. Dentro do reator foram colocadas as células eletrolíticas, formadas por 8 placas de alumínio com $3 \mathrm{~mm}$ de espessura, espaçadas $10 \mathrm{~mm}$ uma da outra, formando uma área superficial total de $400 \mathrm{~cm}^{2}$, sendo a área de cada eletrodo (cátodo e ânodo) de $200 \mathrm{~cm}^{2}$. O conjunto formado possui 2 coletores distribuidores da energia elétrica, um alimentado com carga elétrica positiva (+) e outro com negativa (-), arranjados de forma que cada placa tivesse como vizinha outra placa com carga elétrica oposta, formando um circuito em paralelo (Figura 1).

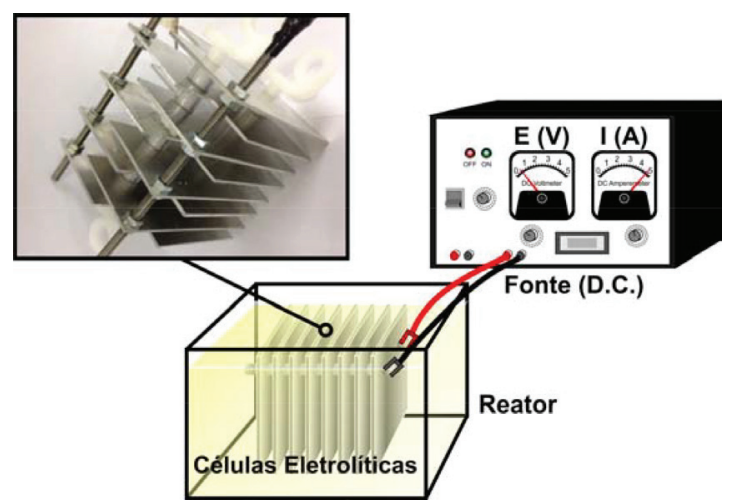

Figura 1. Representação esquemática do sistema eletroquímico de ECF

Para a aplicação da diferença de potencial no reator foi utilizada uma fonte retificadora com potência aparente de $180 \mathrm{~W}$, marca Metron, modelo M-60, com controle de potencial terminal através de um circuito eletrônico chaveado por transistores. O controle do potencial elétrico foi efetuado manualmente através de um potenciômetro, com intervalo de operação de 0 a $24 \mathrm{~V}$. A fonte possui 1 amperímetro e 1 voltímetro utilizados para visualização dos parâmetros de controle.

Para realização dos ensaios, primeiramente, foi fixado um valor para o potencial elétrico aplicado às placas. Isso foi feito através da observação da quantidade de bolhas de gases geradas pela eletrólise para um determinado potencial. Durante esta fase, observou-se a limitação elétrica da fonte retificadora, para evitar o curto-circuito nos componentes eletrônicos da fonte.

Após o potencial elétrico ter sido determinado, o menor tempo necessário para que o efluente fosse totalmente clarificado foi avaliado. No decorrer dessa etapa foram feitos inúmeros ensaios cinéticos para remoção da cor, com intervalos de tempo de 0 a $30 \mathrm{~min}$. Durante todos os ensaios, a cada intervalo de 5 minutos, foi feita a inversão de polaridade dos eletrodos, com o intuito de prevenir a formação de filme passivo.

\section{Tratamento da água residual}

A água residual bruta (efluente líquido) foi coletada na entrada da estação de tratamento de efluentes da empresa, sem nenhum tratamento preliminar. Os ensaios de ECF foram conduzidos em laboratório, utilizando 2,0 L da água residual bruta, inicialmente homogeneizada, variando o tempo de exposição (0-30 min) ao potencial elétrico do reator. Durante o processo ECF o sistema não foi homogeneizado, apenas ocorreu a mistura natural através da formação e purga de bolhas de gases formados durante o processo eletroquímico.

$\mathrm{O}$ ajuste do potencial terminal utilizado foi efetuado com base na corrente elétrica máxima admissível para fonte. A avaliação da eficiência do sistema foi realizada através das análises físico-químicas do efluente bruto e tratado. O monitoramento dos parâmetros e a duração dos experimentos foram feitos de acordo com o estudo cinético realizado.

A melhor condição de tratamento foi selecionada e avaliada por meio de ensaios de cor ( $\mathrm{uC}$ ) e turbidez (uT), utilizando um espectrofotômetro modelo Spectroquant NOVA60 (MERCK). Outros parâmetros também foram analisados, tais como: $\mathrm{pH}$, demanda química de oxigênio (DQO), demanda bioquímica de oxigênio medida a 5 dias (DBO5), óleos e graxas, fósforo e sólidos suspensos.

\section{Análises físico-químicas da água residual bruta e tratada}

As análises físico-químicas para água residual bruta e tratada foram realizadas de acordo os procedimentos do Standard Methods for Examination of Water and Wastewater, 22 Edition. ${ }^{21}$

A determinação de nitrogênio proteico foi realizada através da precipitação das proteínas com ácido tricloroacético (TCA) a $10 \% .^{22}$ Os teores de nitrogênio do precipitado e do sobrenadante resultante foram analisados pelo método de Kjeldahl.

\section{Análise estatística}

Para a comparação entre as médias dos tratamentos, foi utilizado o teste de Tukey a 5\% de probabilidade com nível de confiança igual a $95 \%$.

\section{RESULTADOS E DISCUSSÃO}

\section{Caracterização da água residual}

A caracterização química da água residual bruta foi realizada através das análises de nitrogênio proteico, nitrogênio não proteico, 
nitrogênio total, proteína bruta, matéria orgânica total, fósforo e lipídeos, conforme mostrado na Tabela 1.

Tabela 1. Resultados da caracterização química da água residual do beneficiamento de pescados

\begin{tabular}{lc}
\hline Parâmetros analisados & Resultados obtidos \\
\hline Nitrogênio proteico $\left(\mathrm{mg} \cdot \mathrm{L}^{-1}\right)$ & $12,3 \pm 0,6$ \\
Nitrogênio não proteico $\left(\mathrm{mg} \cdot \mathrm{L}^{-1}\right)$ & $3,2 \pm 0,2$ \\
Nitrogênio total $\left(\mathrm{mg} \cdot \mathrm{L}^{-1}\right)$ & $15,9 \pm 0,7$ \\
Proteína bruta $\left(\mathrm{mg} \cdot \mathrm{L}^{-1}\right)$ & $99,37 \pm 8,0$ \\
Matéria orgânica total $\left(\mathrm{mg} \cdot \mathrm{L}^{-1}\right)$ & $3372 \pm 432$ \\
Lipídeos $\left(\mathrm{mg} \cdot \mathrm{L}^{-1}\right)$ & $113 \pm 16$ \\
Fósforo $\left(\mathrm{mg} \cdot \mathrm{L}^{-1}\right)$ & $6,7 \pm 0,9$ \\
\hline
\end{tabular}

Os resultados das análises mostraram que a água residual bruta apresenta concentrações significativas de nitrogênio proteico $\left(12,3 \mathrm{mg} \mathrm{L}^{-1}\right)$, proteína bruta $\left(99,37 \mathrm{mg} \mathrm{L}^{-1}\right)$, matéria orgânica total $\left(3372 \mathrm{mg} \mathrm{L}^{-1}\right)$, lipídeos $\left(113 \mathrm{mg} \mathrm{L}^{-1}\right)$ e fósforo $\left(6,7 \mathrm{mg} \mathrm{L}^{-1}\right)$. Estes dados foram utilizados para avaliação da composição química $(\mathrm{N}, \mathrm{P}, \mathrm{C})$ da água produzida no beneficiamento de pescados e, assim, definir a metodologia mais adequada para o tratamento. Outros resultados das análises físico-químicas são apresentados na Tabela 3.

\section{Análises de cor e turbidez}

Os melhores resultados para análise da cor e turbidez foram observados com o potencial elétrico em torno de $15 \mathrm{~V}$, distância entre as placas de $10 \mathrm{~mm}$ e corrente de $5 \mathrm{~A}$, com geração expressiva de bolhas no sistema. É importante ressaltar que a corrente desempenha papel fundamental no tamanho e quantidade de bolhas de gás formadas no processo. ${ }^{23}$ Existe uma proporcionalidade entre a a corrente e a quantidade de bolhas. ${ }^{24} \mathrm{Em}$ valores de potencial e distância entre as placas mais baixos não se formaram bolhas suficiente para a flotação das partículas, e em potenciais mais elevados não foi perceptível aumento expressivo na formação de bolhas, justificando, assim, o emprego do potencial de $15 \mathrm{~V}$, corrente de $5 \mathrm{~A}$ e distância entre as placas de $10 \mathrm{~mm}$.

A Figura 2 mostra a diferença entre o efluente no instante de tempo zero (Figura 2a) e após 20 min de tratamento (Figura 2b). Com o tempo superior a $20 \mathrm{~min}$, observou-se que não havia mais alteração da cor da amostra.
A cor para este tipo de efluente é, geralmente, um indicador da presença da matéria orgânica solúvel, oriunda da degradação de matéria de origem animal ou de animais microscópicos em suspensão na água. ${ }^{25} \mathrm{~A}$ cor, normalmente, é de tonalidade amarelada com elevados valores de turbidez, que, por sua vez, reduz a transparência devido à presença de matéria orgânica em suspensão. ${ }^{26,27}$ Grande parte dos microrganismos patogênicos são dissolvidos na presença destas partículas e, por isso, a eliminação delas é fundamental em águas residuais.

Após a definição das condições do sistema, as amostras foram submetidas às análises cinéticas para avaliação dos parâmetros cor, turbidez e variação do $\mathrm{pH}$. Para determinar a cinética de ECF para remoção de cor e turbidez, alíquotas foram retiradas em intervalos de 5 min até completar $30 \mathrm{~min}$, o que possibilitou a construção da curva cinética e, consequentemente, a determinação do tempo de equilíbrio. O tempo de equilíbrio foi atingido quando os valores de cor e turbidez permaneceram constantes. Os resultados obtidos, tanto para cor quanto para turbidez, podem ser observados nas curvas da Figura 3. Observa-se que ocorre uma redução de cerca de $50 \%$ nos primeiros 5 min e que após 25 min de tratamento a água residual bruta ficou completamente clarificada (Figura 3b), apresentando valores próximos a zero para cor e turbidez, independentemente da amostra coletada. $\mathrm{O}$ alto desvio apresentado nos pontos iniciais da curva é devido à grande variabilidade da água residual tratada (5 amostras). Todavia, o sistema de tratamento de ECF demonstrou robustez, pois apesar da variabilidade inicial houve uma redução expressiva dos parâmetros estudados ao final do processo.

Com o transcorrer da ECF percebeu-se um incremento gradual do $\mathrm{pH}$ do meio (Figura 3a), o qual aparentemente se estabiliza no valor de 9. A elevação do $\mathrm{pH}$ do meio é um comportamento esperado no tratamento de águas residuais de caráter levemente ácido (como o originado na indústria de pescado). Outro efeito a ser considerado é que durante a dissolução química do alumínio, íons $\mathrm{H}^{+}$serão consumidos, ocasionando o aumento do $\mathrm{pH}$. Além disso, a reação expressa na Equação (5) será deslocada para direita, promovendo também o incremento no $\mathrm{pH}$ do meio. ${ }^{28}$

$$
\mathrm{Al}_{(a q)}{ }^{3+}+3 \mathrm{H}_{2} \mathrm{O} \rightarrow \mathrm{Al}(\mathrm{OH})_{3(s)}+3 \mathrm{H}_{(a q)}^{+}
$$

Nos instantes finais do processo (25 a $30 \mathrm{~min}$ ), a estabilização no valor do $\mathrm{pH}$ pode estar associada à capacidade tamponante proveniente do tratamento eletroquímico, a qual está intimamente relacionada com o balanço entre a produção e o consumo de $\mathrm{OH}^{-}$, prevenindo grandes oscilações do $\mathrm{pH}$ do sistema. ${ }^{29}$ Esse efeito também foi verificado em outros trabalhos, tais como em estudos quantitativos (a)

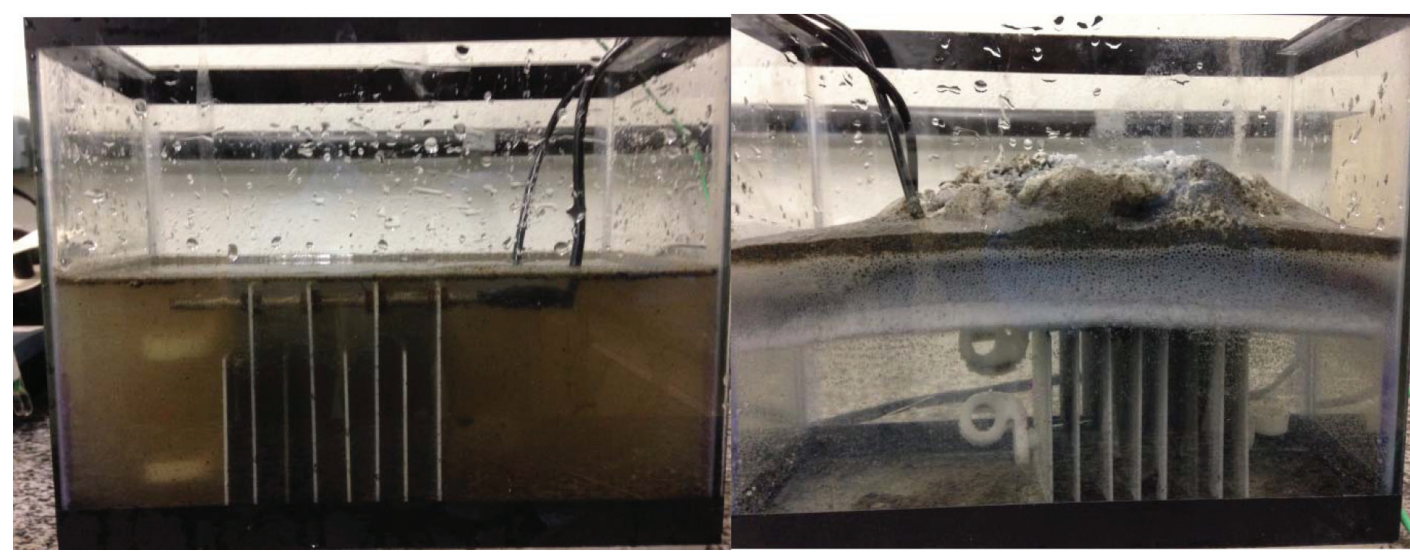

Figura 2. Tratamento por eletrocoagulação-flotação: (a) tempo zero, (b) após 20 minutos 


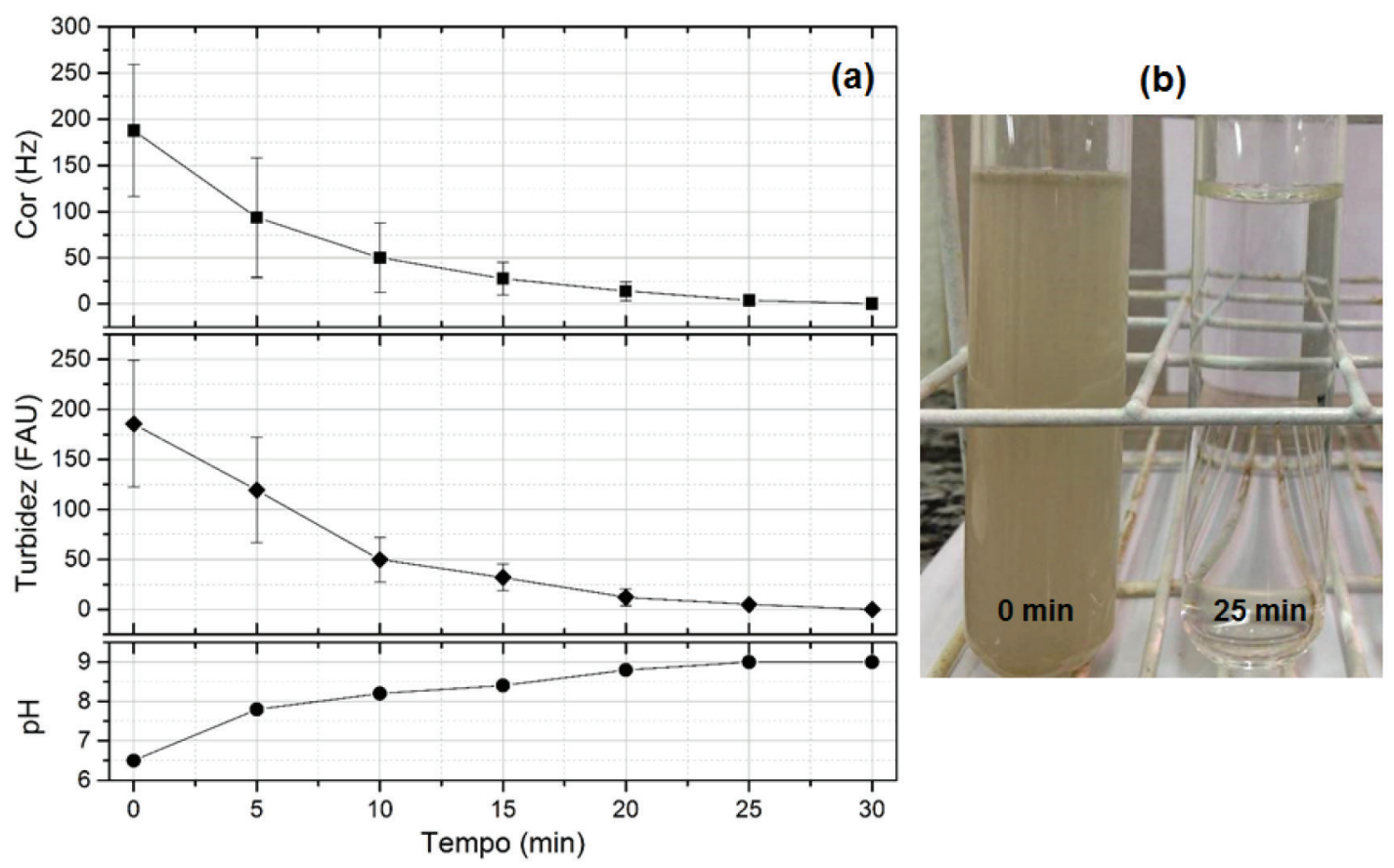

Figura 3. (a) Cinética de variação do pH e da remoção da cor e turbidez da água residual da indústria de pescados (média de 5 experimentos). (b) Amostra da água residual bruta e tratada com corrente de 5 Ampere (após 25 minutos)

comparando a dosagem química e a ECF em tratamento de águas residuais,$^{30}$ em estudos da avaliação do desempenho do processo de eletrocoagulação na remoção da dureza da água potável ${ }^{31} \mathrm{e}$ em estudos do tratamento de soluções sintéticas simulando águas residuais de lavagem contaminada por fluidos de perfuração de poços de petróleo. ${ }^{14}$

\section{Análises físico-químicas}

A avaliação da qualidade da água residual tratada por ECF foi realizada por meio de análises de $\mathrm{pH}$, cor, turbidez, DQO e DBO5 em diferentes correntes. Todas as determinações foram realizadas em triplicata.

De acordo com resultados obtidos, com diferentes potenciais eletroquímicos, foi observado que não ocorreu alteração significativa do $\mathrm{pH}$ para corrente de $1 \mathrm{~A}$ (Tabela 2) em comparação com o efluente bruto. A amostra para 1 A não apresentou bom desempenho no processo, o que pode ser devido à baixa corrente, pois a hidrólise do alumínio depende do potencial aplicado. ${ }^{28}$

Aos resultados obtidos foi aplicado o teste de Tukey. Valores seguidos da mesma letra, na coluna, não diferem estatisticamente entre si a 5\% de probabilidade pelo teste de Tukey (Tabela 2).

O teste de Tukey demonstra claramente que o aumento da corrente favoreceu o tratamento proposto. Resultados promissores foram obtidos com a corrente de $5 \mathrm{~A}$, a qual reduziu significativamente, acima de $90 \%$, quase todos os parâmetros avaliados (cor, turbidez, DQO e DBO5). Embora o pH tenha sido aumentado de maneira significativa (do ponto de vista estatístico), ainda se encontra dentro dos parâmetros legais de descarte ( $\mathrm{pH} 5$ a 9). ${ }^{32}$

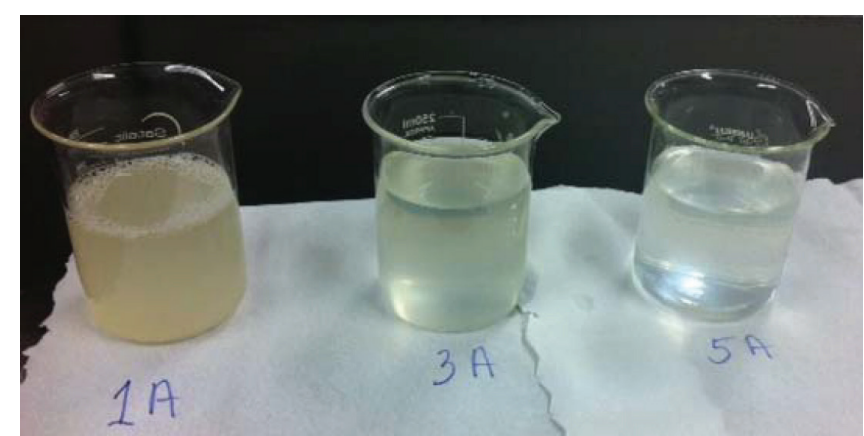

Figura 4. Amostra do efluente tratado com diferentes correntes, 1, 3 e $5 \mathrm{~A}$

Com o intuito de verificar a eficácia do tratamento com corrente de $5 \mathrm{~A}$, foram realizadas cinco repetições com avaliação e caracterização físico-química da água residual bruta e tratada. A Tabela 3 apresenta a média dos resultados, a eficiência de remoção de cada parâmetro analisado e os valores estabelecidos pela legislação brasileira para efeitos de comparação.

Conforme resultados apresentados na Tabela 3, a ECF tem a propriedade de aumentar o $\mathrm{pH}$ do meio, especialmente quando o meio apresenta característica levemente ácida, conforme foi explanado anteriormente.

Os resultados das análises físico-químicas demonstraram uma redução significativa da cor, turbidez, DBO5, DQO, fósforo, sólidos suspensos e óleos e graxas, independentemente da coleta. Isso mostra que o tratamento eletroquímico via ECF é robusto e eficiente.

De modo geral, todos os parâmetros analisados apresentaram

Tabela 2. Resultados obtidos para água residual bruta e tratado utilizando diferentes correntes eletroquímicas, 1, 3 e 5 A

\begin{tabular}{cccccc}
\hline Amostra & $\mathrm{pH}$ & Cor $(\mathrm{Hz})$ & Turbidez $($ FAU $)$ & DQO $\left(\mathrm{mg} \cdot \mathrm{L}^{-1}\right)$ & DBO $\left(\mathrm{mg} \cdot \mathrm{L}^{-1}\right)$ \\
\hline Bruta & $6,5 \pm 0,1^{\mathrm{a}}$ & $182 \pm 50^{\mathrm{a}}$ & $231 \pm 42^{\mathrm{a}}$ & $3466 \pm 234^{\mathrm{a}}$ & $2769 \pm 780^{\mathrm{a}}$ \\
$1 \mathrm{~A}$ & $6,6 \pm 0,1^{\mathrm{a}}$ & $145 \pm 24^{\mathrm{a}}$ & $172 \pm 55^{\mathrm{a}}$ & $2213 \pm 529^{\mathrm{b}}$ & $1812 \pm 298^{\mathrm{a}, \mathrm{b}}$ \\
$3 \mathrm{~A}$ & $7,6 \pm 0,2^{\mathrm{b}}$ & $97 \pm 41^{\mathrm{a}, \mathrm{b}}$ & $56 \pm 8^{\mathrm{b}}$ & $1429 \pm 138^{\mathrm{c}}$ & $1213 \pm 183^{\mathrm{b}}$ \\
$5 \mathrm{~A}$ & $9,0 \pm 0^{\mathrm{c}}$ & $10 \pm 3^{\mathrm{b}}$ & $7 \pm 2^{\mathrm{b}}$ & $293 \pm 19^{\mathrm{d}}$ & $66 \pm 9^{\mathrm{c}}$ \\
\hline
\end{tabular}


Tabela 3. Comparação entre os parâmetros analisados para água residual bruta e tratada por eletrocoagulação-flotação com corrente de 5 A

\begin{tabular}{|c|c|c|c|c|}
\hline Parâmetros & Água Bruto & Água Tratada & Eficiência(\%) & LEGISLAÇÃO ${ }^{\mathrm{a}}$ \\
\hline $\mathrm{pH}$ & $6,5 \pm 0,1$ & $8,8 \pm 0,2$ & - & 5 a 9 \\
\hline Cor $(\mathrm{Hz})$ & $165 \pm 44$ & $8 \pm 2$ & 95,5 & - \\
\hline Turbidez (FAU) & $193 \pm 58$ & $6 \pm 3$ & 96,9 & - \\
\hline $\mathrm{DQO}\left(\mathrm{mg} \cdot \mathrm{L}^{-1}\right)$ & $3372 \pm 432$ & $226 \pm 73$ & 93,3 & - \\
\hline $\mathrm{DBO}\left(\mathrm{mg} . \mathrm{L}^{-1}\right)$ & $2433 \pm 417$ & $62 \pm 10$ & 97,5 & Remoção de $60 \%$ \\
\hline Fósforo $\left(m g . L^{-1}\right)$ & $6,7 \pm 0,9$ & $0,8 \pm 0,3$ & 88,5 & - \\
\hline Sólidos Susp. (mg.L $\mathrm{L}^{-1}$ ) & $177 \pm 35$ & N.D. & 100,0 & - \\
\hline Óleos e Graxas (mg.L-1) & $113 \pm 16$ & $7 \pm 2$ & 93,6 & $50 \mathrm{mg} \cdot \mathrm{L}^{-1}$ \\
\hline Alumínio (mg.L-1) & N.D. & 5,5 & - & - \\
\hline
\end{tabular}

${ }^{a}$ CONAMA No $430 / 2011$.

uma eficiência média acima de $90 \%$ na remoção dos contaminantes, sendo as maiores reduções observadas na remoção da cor, turbidez e DBO5, com 95,5; 96,9 e 97,5\%, respectivamente. Entretanto, o teor de alumínio na água tratada, decorrente do desgaste do eletrodo durante o processo de ECF, foi $5,5 \mathrm{mg} \mathrm{L}^{-1}$. É importante ressaltar que a legislação brasileira não estabelece limites para alumínio em efluentes. ${ }^{32}$

Valores semelhantes também foram observados em outros trabalhos, utilizando a ECF na separação de poluentes das águas residuais de restaurantes, ${ }^{33}$ e no tratamento de águas residuais de lavagem. ${ }^{34}$

Os resultados também mostraram que uma das maiores vantagens da ECF é na remoção de óleos e graxas, com remoção média acima de $93 \%$. Esse fato ocorre devido a uma melhor interação dessas moléculas com o hidróxido de alumínio, facilitando, assim, a coagulação e a flotação dessas moléculas através da formação de coloides cuja densidade é menor que a da água. ${ }^{35}$ Esses coloides deslocam-se naturalmente para a superfície da câmara de separação. No entanto, a flotação das partículas coloidais é mais rápida e eficiente por causa da formação das microbolhas geradas no processo. ${ }^{36}$

Essa peculiaridade da ECF pode ser aproveitada para utilização no tratamento de efluentes contaminados com altas concentrações de óleos e graxas. Diversos trabalhos na literatura demonstraram a aplicação do processo de ECF no tratamento de águas residuais com elevados teores de óleos e graxas, ${ }^{29}$ no tratamento de águas residuais oleosas do moinho de azeite, ${ }^{37}$ na avaliação do efeito da natureza do eletrólito no tratamento de soluções óleosas por $\mathrm{ECF}^{38}$ e na remoção de óleos e graxas de um reservatório de água residual. ${ }^{39}$

Além disso, o processo de ECF tem duas vantagens significativas, menor tempo de retenção e menor quantidade de lodo produzido quando comparado com os processos convencionais. Nestas condições, a quantidade de lodo seco foi de $0,35-0,40 \mathrm{~kg}_{\text {por }} \mathrm{m}^{3}$ de água residual tratada. Este valor corresponde apenas 35 a $40 \%$ do lodo total produzido por processos convencionais de coagulação/flotação utilizando aditivos químicos. Entretanto, a concentração de alumínio residual no lodo seco foi de $8,5 \%$, matéria orgânica $55,06 \%$, cinzas $35,50 \%$ e proteína bruta $20,50 \mathrm{mg} \mathrm{kg}^{-1}$, como mostra a Tabela 4.

É válido mencionar que existem poucos trabalhos na literatura sobre a caracterização do lodo gerado no processo de tratamento de água residual por ECF. No entanto, de acordo com os critérios de classificação de resíduos sólidos da NBR 10004/2004, o lodo desidratado (seco) é um resíduo não perigoso, classe II. Este resíduo desidratado pode ser aproveitado como combustível para fornalhas, e as cinzas geradas na combustão podem ser misturadas com o barro para fazer tijolos e/ou cerâmicas comerciais. A argilas têm em sua composição química: alumina, sílica, óxidos de ferro, magnésio, calcário, componentes esses que aumentam a resistência ao calor e melhoram a resistência a compressão.
Tabela 4. Caracterização do lodo seco formado no processo de eletrocoagulação-flotação da água residual do beneficiamento de pescados

\begin{tabular}{lc}
\hline Parâmetros analisados & Resultados obtidos \\
\hline Alumínio (\%) & $8,5 \pm 0,3$ \\
Matéria orgânica total (\%) & $55,06 \pm 0,4$ \\
Cinzas (\%) & $35,5 \pm 0,4$ \\
Nitrogênio total (mg.kg-1) & $3,2 \pm 0,2$ \\
Proteína bruta (mg.kg-1) & $20,5 \pm 2,0$ \\
\hline
\end{tabular}

Outros estudos relevantes envolvendo ECF também são descritos na literatura, como, por exemplo, na remoção de uma variedade de partículas dissolvidas, matéria orgânica em suspensão, íons de dureza de água, nitrato e fosfato. ${ }^{40}$ Os resultados mostraram que a remoção desses íons justifica os efeitos da desinfecção produzidos pelo processo de ECF. Com este estudo foi verificado que a remoção de bactérias e algas pode ser alcançada em $30 \mathrm{~min}$. Com isso a ECF permitiria limitar o uso de biocidas e, consequentemente, reduzir custos nas estações de tratamento de água. A ECF também foi aplicada no tratamento de águas residuais de lavanderia, no qual foi observado remoção significativa (acima de 90\%) da turbidez, DQO, COD, fosfato e MBAS, simultaneamente..$^{28}$ Bons resultados também foram obtidos em escala piloto no tratamento de águas residuais de restaurante, ${ }^{41}$ na remoção de metais não ferrosos e metais pesados moderadamente solúveis em água ${ }^{42}$ e na separação da biomassa de microalgas utilizando eletrodos de ferro e alumínio. ${ }^{43}$

A fim de estudar a robustez do tratamento aplicado, foi coletado efluente de outra empresa de beneficiamento de pescados, com características diferentes, pois esta empresa trabalha somente com processamento de peixes. Os ensaios eletroquímicos foram realizados nas mesmas condições. Após o tratamento, as amostras tratadas foram submetidas às análises físico-químicas, e os resultados com as porcentagens de remoção são mostrados na Figura 5. Conforme pode ser observado, os resultados na eficiência da remoção dos contaminantes foram similares para sólidos suspensos, DBO5, cor e turbidez. Entretanto, para os parâmetros de DQO (78\%), fósforo (60\%) e óleos e graxas (83\%), os resultados foram menores.

Os resultados obtidos demonstraram que o tratamento com o reator eletroquímico de ECF, nesta empresa, promoveu uma redução significativa em todos os parâmetros analisados, com remoção média acima de $90 \%$ para DBO5; 95,6\% e 94,9\% para cor e turbidez, respectivamente. A grande redução no teor de matéria orgânica pode ser relacionada à oxidação das substâncias orgânicas dissolvidas, a qual se deve à presença das pequenas bolhas de oxigênio que atuam como 


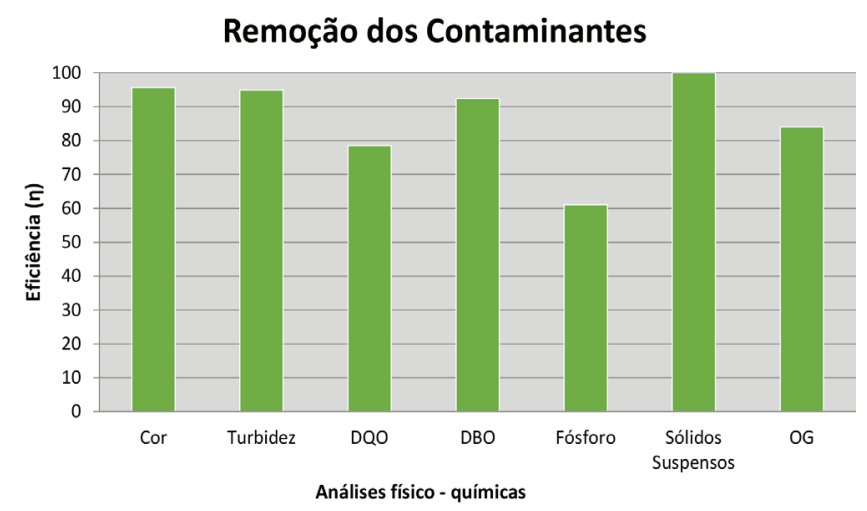

Figura 5. Eficiência de remoção dos contaminantes por ECF, corrente de 5 A, tempo de $25 \mathrm{~min}$

um potente agente oxidante no meio. ${ }^{12}$ Isso de fato confirma o potencial da técnica empregada para o tratamento do efluente em questão.

\section{CONCLUSÃO}

A técnica eletroquímica de ECF vem ganhando popularidade e se tornando uma alternativa reconhecidamente viável e eficiente diante dos processos convencionais para o tratamento de águas residuais. De acordo com resultados obtidos, pode-se concluir que a ECF é um processo com grande potencial para o tratamento de águas residuais provenientes da indústria de beneficiamento de pescados.

A melhor condição estudada (corrente de $5 \mathrm{~A}$, potencial elétrico de $15 \mathrm{~V}$ e distância entre as placas igual a $10 \mathrm{~mm}$ ) apresentou uma eficiência de remoção dos contaminantes bastante significativa. Para a turbidez ocorreu uma redução média de 96,9\%; DQO de 93,3; DBO5 de 97,5\%; fósforo de $88,5 \%$ e óleos e graxas de $93,6 \%$. No que diz respeito à cor, observou-se que ocorreu uma redução acima de $50 \%$ nos 5 primeiros minutos e após 25 min de tratamento a água residual foi completamente clarificada (redução média acima de 95\%). Além disso, o processo de ECF apresenta algumas vantagens quando comparado aos processos convencionais, como o menor tempo de retenção, menor área de instalação e ainda uma menor produção de lodo no final do tratamento.

\section{AGRADECIMENTOS}

Os autores agradecem à UNISUL (Universidade do Sul de Santa Catarina), à FAPESC (Fundação de Amparo à Pesquisa e Inovação do Estado de Santa Catarina) e ao CNPq (Conselho Nacional de Desenvolvimento Científico e Tecnológico) pelo apoio financeiro.

\section{REFERÊNCIAS}

1. Bottino, A.; Capannelli, G.; Comite, A.; Ferrari, F.; Firpo, R.; Venzano, S.; C. R. Chimie 2009, 12, 882.

2. Sivasangari, S.; Suseendhar, S.; Kumar, K. S.; Vijayaprasath, N.; Thirumurugan, M.; International Journal of Innovative Research in Science, Engineering and Technology 2016, 5, 20810.

3. Mollah, M. Y. A.; Schennach, R.; Parga, J. R.; Cocke, D. L.; J. Hazard. Mater. 2001, 84, 29.

4. Nawel, A.; Farid, D.; Belkacem, M.; Jean-Pierre, L.; Khodir, M.; Sep. Purif. Technol. 2015, 144, 168.

5. Tikariha, A.; Sahu, O.; J. Appl. Environ. Microbiol. 2014, 2, 16.
6. Colic, M.; Morse, W.; Hicks, J.; Lechter, A.; Miller, J. D.; Industrial Water Quality 2007, 27, 1.

7. Durai, G.; Rajasimman, M.; J. Environ. Sci.Technol. 2011, 4, 1.

8. Angelis, D. F.; Corso, C. R.; Vidoia, E.; Quim. Nova 1998, 20, 24.

9. Afonso, M. D.; Bórquez, R.; Desalination 2002, 142, 29.

10. Chowdhury, P.; Viraraghavan, T.; Srinivasan, A.; Bioresour. Technol. 2010, 101, 439.

11. Lefebvre, O.; Moletta, R.; Water Res. 2006, 40, 3671.

12. Mansour, L. B.; Kesentini, I.; J. Hazard. Mater. 2008, 153, 1067.

13. Comninellis, C.; Electrochim. Acta 1994, 39, 857.

14. Mota, I. O.; Castro, J. A.; Casqueira, R. G.; Junior, A. G. O.; J. Mater. Res. Technol. 2015, 4, 109.

15. Cerqueira, A. A.; Marques, M. R. C.; Russo, C.; Quim. Nova 2011, 34, 59.

16. Kyzas, G. Z.; Matis, K. A.; J. Mol. Liq. 2016, $220,657$.

17. Sahu, O.; Mazumdar, B.; Chaudhari, P. K.; Environ Sci Pollut Res. 2014, 21, 2397.

18. Khelifa, A.; Aoudj, S.; Moulay, S.; Petris-Wery, M.; Chem. Eng. Process. 2013, 70, 110.

19. ABNT - NBR 9897: Planejamento de amostragem de efluentes líquidos e corpos receptores, 1987.

20. ABNT - NBR 9898: Preservação e técnicas de amostragem de efluentes líquidos e corpos receptores, 1987.

21. APHA; Standard Methods for the Examination of Water and Wastewater, $22^{\text {th }}$ ed., American Public Health Association: New York, 2012.

22. Tshinyangu, K. K.; Hennebert, G. L.; Food Chem. 1996, 57, 223.

23. Fukui, Y.; Yuu, S.; AlChE Journal 1985, 31, 201.

24. Chen, G.; Sep. Purif. Technol. 2004, 38, 11.

25. Chowdhury, P.; Viraraghavan, T.; Srinivasan, A.; Bioresour. Technol. 2010, 101, 439.

26. Afonso, M. D.; Bórquez, R.; Desalination 2002, 142, 29.

27. Wong, Y.C.; Moganaragi, V.; Atiqah, N.A.; Orient. J. Chem. 2013, 29, 1421.

28. Ge, J.; Qu, J.; Lei, P.; Liu, H.; Sep. Purif. Technol. 2004, 36, 33.

29. Chen, G.; Sep. Purif. Technol. 2004, 38, 11.

30. Holt, P. K.; Barton, G. W.; Wark, M.; Mitchell, C. A.; Colloids Surf., A 2002, 211, 233.

31. Malakootian, M.; Mansoorian, H. J.; Moosazadeh, M.; Desalination 2010, 255, 67.

32. CONAMA; Resolução $n^{\circ} 430,13$ de maio de 2011.

33. Chen, X.; Chen, G.; Yue, P. L.; Sep. Purif. Technol. 2000, 19, 65.

34. Wang, C.; Choub, W.; Kuo, Y.; J. Hazard. Mater. 2009, 164, 81.

35. Mollah, M. Y. A.; Morkovskyb, P.; Gomes, J. A. G.; Kesmez, M.; Parga, J.; Cocke, D. L.; J. Hazard. Mater. 2004, 144, 199.

36. Llerena, C.; Ho, J. C. K.; Piron, D. L.; Chem. Eng. Commun. 1996, 155, 217.

37. Inan, H.; Dimoglo, A.; Simsek, H.; Karpuzcu, M.; Sep. Purif. Technol. 2004, 36, 23.

38. Izquierdo, C. J.; Canizares, P.; Rodrigo, M.A.; Leclerc, J.P.; Valentin, G.; Lapicque, F.; Desalination 2010, 255, 15.

39. Ji, M.; Jiang, X.; Wang, F.; Desalin. Water Treat. 2015, 55, 2044.

40. Ricordel, C.; Darchenb, A.; Hadjievc, D.; Sep. Purif. Technol. 2010, 74, 342.

41. Qin, X.; Yang, B.; Gao, F.; Chen, G.; J. Environ. Eng. 2013, 139, 1004.

42. Il'in, V. I.; Brodskii, V. A.; Kolesnikov, V. A.; Gubin, A. F.; Russ. J. Gen. Chem. 2014, 84, 2332.

43. Baierle, F.; John, D. K.; Souza, M. P.; Bjerk, T. R.; Moraes, M. S. A.; Hoeltz, M.; Rohlfes, A. L. B.; Camargo, M. E.; Corbellini, V. A.; Schneider, R. C. S.; Chem. Eng. J. 2015, 267, 274. 